\title{
A Fast Algorithm for Linear Estimation of Two-Dimensional Isotropic Random Fields
}

\author{
BERNARD C. LEVY, MEMBER, IEEE, AND JOHN N. TSITSIKLIS, MEMBER, IEEE
}

\begin{abstract}
The problem considered involves estimating a two-dimensional isotropic random field given noisy observations of this field over a disk of finite radius. By expanding the field and observations in Fourier series, and exploiting the covariance structure of the resulting Fourier coefficient processes, recursions are obtained for efficiently constructing the linear least-squares estimate of the field as the radius of the observation disk increases. These recursions are similar to the Levinson equations of one-dimensional linear prediction. In the spectral domain they take the form of Schrödinger equations, which are used to give an inverse spectral interpretation of our estimation procedure.
\end{abstract}

\section{INTRODUCTION}

$\mathrm{T}$ HE OBJECTIVE of this paper is to extend the Levinson recursions of one-dimensional (1-D) linear prediction [1], [2] to the problem of estimating a two-dimensional (2-D) isotropic random field given noisy observations of this field over a disk of finite radius. By expanding the field and observations in Fourier series and by exploiting the covariance structure of the resulting coefficient processes, some wavelike equations will be obtained for solving the filtcring problem associated with the coefficient processes. These equations will be used to estimate the field and to compute the optimum filter recursively as the radius of the observation disk increases.

The wavelike equations satisfied by the optimum filters for the Fourier coefficient processes can be transformed into coupled first-order equations that, when discretized, require considerably fewer operations than standard nonrecursive methods [3]-[5] for estimating isotropic random fields. This is the first fast algorithm to be derived for estimating 2-D random fields, and its complexity is the same as that of the 1-D Levinson recursions.

By transforming the estimation problem over a finite disk into an equivalent problem in the spectral domain, the wave equations that we obtain are transformed into Schrödinger equations with a circularly symmetric potential. These equations constitute the 2-D counterpart of the recursions satisfied by the 1-D Krein polynomials [6], [7]. A simple expression for recursively computing the potentials appearing in these Schrödinger equations is given, and

Manuscript received July 11, 1984. This work was supported by the National Science Foundation under Grant ECS-83-12921, the Air Force Office of Scientific Research under Grant AFOSR-82-0135B, and the Office of Naval Research under Grant ONR/00014-77-C-0532 (NR 041-519).

The authors are with the Laboratory for Information and Decision Systems and the Department of Electrical Engineering and Computer Science, Massachusetts institute of Technology, Cambridge, MA U2139, USA. an inverse spectral interpretation of our estimation procedure is obtained.

This paper is organized as follows. In Section II the observed field is expanded in Fourier series and some 1-D filtering problems for the Fourier coefficient processes are introduced. These filtering problems are solved by exploiting the structure of the covariance of the coefficient processes, and in Section III this solution is used to estimate an arbitrary random variable. The wavelike equations that we obtain in Section II are transformed into an equivalent set of first-order equations in Section IV, and a recursive numerical scheme is presented. In Section V, the 2-D estimation problem is transformed to the spectral domain, and the wave equations for the Fourier coefficient estimation problem are transformed into Schrödinger equations. These are then used to give an inverse spectral interpretation of our estimation method.

\section{Fourier Coefficient Processes}

Let

$$
y(\boldsymbol{r})=z(\boldsymbol{r})+v(\boldsymbol{r})
$$

with $r \in \mathbb{R}^{2}$, be some noisy observations of a real twodimensional isotropic zero-mean Gaussian random field $z(\cdot)$ with covariance

$$
E[z(\boldsymbol{r}) z(s)]=k(l),
$$

where $l=|\boldsymbol{r}-\boldsymbol{s}|$ denotes the length of the vector $\boldsymbol{r}-\boldsymbol{s}$. Here $v(\cdot)$ is a two-dimensional white Gaussian noise field with unit intensity, i.e.,

$$
E[v(\boldsymbol{r}) v(s)]=\delta(\boldsymbol{r}-s)=\frac{1}{\pi l} \delta(l),
$$

where $\delta(\boldsymbol{r})$ denotes the delta function over $\mathbb{R}^{2}$. We assume that $z(\cdot)$ and $v(\cdot)$ are uncorrelated, so that

$$
E[z(\boldsymbol{r}) v(\boldsymbol{s})] \equiv 0,
$$

and that $k(\cdot) \in L_{1}\left(\mathbb{R}^{+}, l d l\right)$, where $\mathbb{R}^{+}$denotes the positive half-line. In this case $z(\cdot)$ has the spectral density [5], [8], [9]

$$
\hat{k}(\lambda)=\int_{0}^{\infty} J_{0}(\lambda l) k(l) l d l,
$$

where $J_{0}(\cdot)$ denotes the Bessel function of other zero, and

$$
k(l)=\int_{0}^{\infty} J_{0}(\lambda l) \hat{k}(\lambda) \lambda d \lambda .
$$


It will be assumed in this paper that we observe $y(\boldsymbol{r})$ over the disk $D_{R}$ of radius $R$ centered at the origin. Then, if $\boldsymbol{a}$ is an arbitrary random variable in the Hilbert space $Z$ spanned by $z(\boldsymbol{r})$ for $r \in \mathbb{R}^{2}$, the estimation problem that we consider is the one of computing the conditional mean $E\left[a \mid Y_{R}\right]$, where $Y_{R}$ denotes the Hilbert space spanned by the observations $y(\boldsymbol{r})$ for $0 \leq r=|\boldsymbol{r}| \leq R$. The space $Z$ is obtained by taking the mean-square limit of linear combinations of $z(\boldsymbol{r})$ for $\boldsymbol{r} \in \mathbb{R}^{2}$, and elements of $Y_{R}$ are of the form

$$
\begin{aligned}
\boldsymbol{b} & =\int_{D_{R}} b(\boldsymbol{r}) y(\boldsymbol{r}) d \boldsymbol{r} \\
& =\int_{0}^{R} \int_{0}^{2 \pi} b(r, \theta) y(r, \theta) r d r d \theta,
\end{aligned}
$$

where $(r, \theta)$ are the polar coordinates of $\boldsymbol{r}, \mathrm{d} \boldsymbol{r}=\mathrm{rd} \mathrm{rd} \theta$ is an element of area, and $b(\boldsymbol{r})=b(r, \theta) \in L_{2}\left(D_{\mathrm{R}}\right)$.

\section{A. Fourier Series Expansion}

Our estimation procedure will rely on the Fourier series expansion

$$
\begin{aligned}
& y(r, \theta)=\sum_{n=-\infty}^{\infty} y_{n}(r) \exp j n \theta \\
& z(r, \theta)=\sum_{n=-\infty}^{\infty} z_{n}(r) \exp j n \theta \\
& v(r, \theta)=\sum_{n=-\infty}^{\infty} v_{n}(r) \exp j n \theta
\end{aligned}
$$

of the observation, signal, and noise fields, where the Fourier coefficients

$$
\begin{aligned}
& y_{n}(r)=\frac{1}{2 \pi} \int_{0}^{2 \pi} y(r, \theta) \exp (-j n \theta) d \theta \\
& z_{n}(r)=\frac{1}{2 \pi} \int_{0}^{2 \pi} z(r, \theta) \exp (-j n \theta) d \theta \\
& v_{n}(r)=\frac{1}{2 \pi} \int_{0}^{2 \pi} v(r, \theta) \exp (-j n \theta) d \theta
\end{aligned}
$$

are one-dimensional processes, and where

$$
y_{n}(r)=z_{n}(r)+v_{n}(r), \quad 0 \leq r \leq R
$$

leads to the usual linear least-squares filtering problem for $z_{n}$ given $y_{n}$.

The main feature of this expansion is that the Fourier coefficient processes of different orders are uncorrelated, i.e.,

$$
\begin{aligned}
E\left[z_{n}(r) z_{m}^{*}(s)\right] & =E\left[v_{n}(r) v_{m}^{*}(s)\right] \\
& =E\left[z_{n}(r) v_{m}^{*}(s)\right] \equiv 0
\end{aligned}
$$

for $n \neq m$ (see [5, p. 7] and [9]). Consequently, if we denote by $Z^{n}=H\left(z_{n}(r), 0 \leq r \leq \infty\right)$ and $Y_{R}^{n}=H\left(y_{n}(r)\right.$, $0 \leq r \leq R$ ) the Hilbert spaces spanned by the $n^{\text {th }}$ Fourier coefficient signals and observations, the signal and ob- servation spaces can be decomposed as

$$
\begin{gathered}
Z=\bigoplus_{\substack{n=-\infty \\
\prod_{R}}} Z^{n} \\
Y_{n=-\infty}^{\infty} Y_{R}^{n} .
\end{gathered}
$$

To interpret (2.11) the elements of $Z$ or $Y_{R}$ must be viewed as the mean-square limit of finite linear combinations of elements of $Z^{n}$ or $Y_{R}^{n}$ for different values of $n$. Using (2.11) the conditional mean of $z(r, \theta)$ given $Y_{R}$ can be expressed as

$$
E\left[z(r, \theta) \mid Y_{R}\right]=\sum_{n=-\infty}^{\infty} E\left[z_{n}(r) \mid Y_{R}^{n}\right] \exp j n \theta,
$$

so that our original two-dimensional estimation problem requires only the solution of the sequence of one-dimensional estimation problems for the observation equations (2.9).

To solve the estimation problem (2.9), we will exploit the covariance structure of the Fourier coefficient signal and noise processes $z_{n}(\cdot)$ and $v_{n}(\cdot)$. The covariance of $z_{n}(\cdot)$ is given by

$$
\begin{aligned}
k_{n}(r, s) & =E\left[z_{n}(r) z_{n}^{*}(s)\right] \\
& =\int_{0}^{\infty} J_{n}(\lambda r) J_{n}(\lambda s) \hat{k}(\lambda) \lambda d \lambda
\end{aligned}
$$

(see [9]), where $J_{n}(\cdot)$ denotes the Bessel function of order $n$; and $v_{n}(\cdot)$ is a white noise process uncorrclated with $z_{n}(\cdot)$ and with intensity

$$
E\left[v_{n}(r) v_{n}^{*}(s)\right]=\frac{1}{2 \pi r} \delta(r-s) .
$$

Thus $z_{n}(\cdot)$ and $v_{n}(\cdot)$ are not stationary. However, the expression (2.13) for the covariance kernel $k_{n}(\cdot, \cdot)$ indicates that $z_{n}(\cdot)$ has as much structure as a stationary process. This structure can be characterized as follows.

Lemma 1 (Displacement Property of $k_{n}$ ): If $k(\cdot)$ is twice differentiable, or, equivalently, if the field $z(\cdot)$ is meansquare differentiable, we have

$$
\begin{aligned}
\left(\left(\frac{\partial^{2}}{\partial r^{2}}+\right.\right. & \left.\frac{1}{r} \frac{\partial}{\partial r}-\frac{n^{2}}{r^{2}}\right) \\
& \left.\times-\left(\frac{\partial^{2}}{\partial s^{2}}+\frac{1}{s} \frac{\partial}{\partial s}-\frac{n^{2}}{s^{2}}\right)\right) k_{n}(r, s) \equiv 0
\end{aligned}
$$

with the boundary conditions

$$
\frac{\partial}{\partial r} k_{0}(0, s)=0 \text { and } k_{n}(0, s)=0, \quad \text { for } n \neq 0 .
$$

Proof: It is shown in [10, Theorem 2.2.2] that $z(\cdot)$ is mean-square differentiable if and only if $k(\cdot)$ is twice differentiable. Furthermore, since $k(\cdot)$ is twice differentiable, $k_{n}(\cdot, \cdot)$ is twice differentiable. By noting that $J_{n}(\lambda r)$ 
satisfies the differential equation

$$
\left(\frac{d^{2}}{d r^{2}}+\frac{1}{r} \frac{d}{d r}+\left(\lambda^{2}-\frac{n^{2}}{r^{2}}\right)\right) J_{n}(\lambda r)=0
$$

we obtain

$$
\begin{aligned}
\left(\frac{\partial^{2}}{\partial r^{2}}\right. & \left.+\frac{1}{r} \frac{\partial}{\partial r}-\frac{n^{2}}{r^{2}}\right) k_{n}(r, s) \\
& =-\int_{0}^{\infty} \lambda^{2} J_{n}(\lambda r) J_{n}(\lambda s) \hat{k}(\lambda) \lambda d \lambda \\
& =\left(\frac{\partial^{2}}{\partial s^{2}}+\frac{1}{s} \frac{\partial}{\partial s}-\frac{n^{2}}{s^{2}}\right) k_{n}(r, s)
\end{aligned}
$$

So that $(2.15)$ is satisfied. The boundary conditions $(2.16)$ are a consequence of the initial conditions $\dot{J}_{0}(0)=0$ and $J_{n}(0)=0$ for $n \neq 0$.

The displacement property $(2.15)$ is similar to the property

$$
\left(\frac{\partial}{\partial r}+\frac{\partial}{\partial s}\right) k(r, s)=0
$$

of the covariance $k(r, s)=k(r-s)$ of a stationary process. In [7] and [11] this property was used to derive the Levinson recursions for estimating a stationary process. We will see below that (2.15) plays the same role for estimating $z_{n}(\cdot)$.

It will later be useful to consider the normalized estimation problem

$$
\bar{y}_{n}(r)=\bar{z}_{n}(r)+\bar{v}_{n}(r), \quad 0 \leq r \leq R,
$$

where $\bar{y}_{n}(r)=r^{1 / 2} y_{n}(r), \bar{z}_{n}(r)=r^{1 / 2} z_{n}(r)$, and $\bar{v}_{n}(r)=$ $r^{1 / 2} v_{n}(r)$ and where the noise process $\bar{v}_{n}(\cdot)$ is stationary, i.e.,

$$
E\left[\bar{v}_{n}(r) \bar{v}_{n}(s)\right]=\frac{1}{2 \pi} \delta(r-s) .
$$

Then, the covariance of $\bar{z}_{n}(\cdot)$ is given by

$$
\bar{k}_{n}(r, s)=E\left[\bar{z}_{n}(r) \bar{z}_{n}(s)\right]=(r s)^{1 / 2} k_{n}(r, s),
$$

and the displacement property (2.15) takes the form

$$
\begin{aligned}
\left(\left(\frac{\partial^{2}}{\partial r^{2}}-\frac{1}{r^{2}}\left(n^{2}-\frac{1}{4}\right)\right)\right. \\
\left.\quad-\left(\frac{\partial^{2}}{\partial s^{2}}-\frac{1}{s^{2}}\left(n^{2}-\frac{1}{4}\right)\right)\right) \bar{k}_{n}(r, s)=0 .
\end{aligned}
$$

\section{B. Filtered Estimates for the Fourier Coefficients.}

The first step to solve the estimation problem (2.9) is to compute the filtered estimate

$$
E\left[z_{n}(R) \mid Y_{R}^{n}\right]=\hat{z}_{n}(R \mid R)=\int_{0}^{R} g_{n}(R, s) y_{n}(s) s d s
$$

By denoting the filtering error as

$$
\tilde{z}_{n}(R \mid R)=z_{n}(R)-\hat{z}_{n}(R \mid R),
$$

and using the orthogonality property, $\tilde{z}_{n}(R \mid R) \perp Y_{R}^{n}$, of linear least-squares estimates, we find that the filter $g_{n}(\cdot, \cdot)$ satisfies the integral equation

$$
k_{n}(r, R)=\int_{0}^{R} k_{n}(r, s) g_{n}(R, s) s d s+\frac{1}{2 \pi} g_{n}(R, r)
$$

for $0 \leq r \leq R$

To show that the solution of (2.21) exists and is unique, we assume that $k_{n}(\cdot, \cdot) \in L_{2}\left(r s d r d s,[0, R]^{2}\right)$, or, equivalently, that $k(\cdot) \in L_{2}(r d r,[0, R])$. Then, the operator

$$
K_{n}: a(r) \rightarrow b_{n}(r)=\int_{0}^{R} k_{n}(r, s) a(s) s d s
$$

is defined over $L_{2}(r d r,[0, R])$, and since $k_{n}(\cdot, \cdot)$ is a covariance kernel, $\boldsymbol{K}_{n}$ is self-adjoint and nonnegative definite, so that $K_{n}+I / 2 \pi$ is invertible. This guarantees the existence and unicity of a solution in $L_{2}(r d r,[0, R])$ to the integral equation (2.21).

Our method of computing $g_{n}(R, \cdot)$ is to divide the interval $[0, R]$ into $N$ subintervals of length $\Delta=R / N$ and to discretize (2.21) accordingly. The solution of the resulting system of linear equations requires $O\left(N^{3}\right)$ operations, but by exploiting the displacement property of $k_{n}$, we can obtain a more efficient procedure.

Theorem 1: If $k(\cdot)$ is twice differentiable, or, equivalently, if $z(\cdot)$ is mean-square differentiable, and if

$$
\delta_{n}=\left(\frac{\partial^{2}}{\partial R^{2}}+\frac{1}{R} \frac{\partial}{\partial R}-\frac{n^{2}}{R^{2}}\right)-\left(\frac{\partial^{2}}{\partial r^{2}}+\frac{1}{r} \frac{\partial}{\partial r}-\frac{n^{2}}{r^{2}}\right)
$$

denotes the displacement operator used to characterize the structure of $k_{n}(\cdot, \cdot)$, then $g_{n}(\cdot \cdot)$ satisfies the partial differential equation

$$
\delta_{n} g_{n}(R, r)=V_{n}(R) g_{n}(R, r),
$$

where $V_{n}(\cdot)$ is given by

$$
V_{n}(R)=-2 \frac{d}{d R}\left(R g_{n}(R, R)\right)
$$

and where the boundary value $g_{n}(R, R)$ can be found from the relation

$$
g_{n}(R, R)=2 \pi\left(k_{n}(R, R)-\int_{0}^{R} k_{n}(R, s) g_{n}(R, s) s d s\right),
$$

which is obtained by setting $r=R$ in (2.21). In addition, $g_{n}(R, \cdot)$ satisfies the boundary condition

$$
\frac{\partial}{\partial r} g_{0}(R, 0)=0 \quad g_{n}(R, 0)=0, \quad \text { for } n \neq 0
$$

Proof: Operate with $\boldsymbol{\delta}_{n}$ on both sides of (2.21) and use the displacement property (2.15). Integrating by parts gives $V_{n}(R) k_{n}(r, R)$

$$
=\int_{0}^{R} k_{n}(r, s) \boldsymbol{\delta}_{n} g_{n}(R, s) s d s+\frac{1}{2 \pi} \boldsymbol{\delta}_{n} g_{n}(R, r),
$$


where $V_{n}(\cdot)$ is defined by (2.23). Then, by linearity, and since the solution of (2.21) is unique, we obtain (2.22). The boundary conditions (2.25) are a consequence of the conditions (2.16) for $k_{n}(\cdot, \cdot)$.

The hyperbolic partial differential equation (2.22) has a structure similar to that of the continuous-time Levinson recursions [7]. The only difference is that on the left side of (2.22) we have used $\delta_{n}$ for displacement operator, instead of

$$
\tau=\frac{\partial}{\partial R}+\frac{\partial}{\partial r}
$$

for the Levinson recursions. Equation (2.22) shows that the optimum filter $g_{n}(\cdot, \cdot)$, which is a function of two variables, is parametrized by $V_{n}(\cdot)$, which depends on one variable only. Note that $V_{n}(R)$ is given as a function of the boundary value $g_{n}(R, R)$, so the partial differential equation (2.22) is nonlinear. The given data, which is the covariance $k_{n}(\cdot, \cdot)$, is introduced in equation (2.22) via relation (2.24), which is used to evaluate the bounday value $g_{n}(R, R)$ and then to compute $V_{n}(R)$ through (2.23). To compute $g_{n}(R, r)$ over the triangle $0 \leq r \leq R$, a finite difference discretization scheme can be applied to (2.22), or to an equivalent system of first-order equations for $(\partial / \partial R) g_{n}(R, r)$ and $(\partial / \partial r) g_{n}(R, r)$, as shown in [12, Chapter 6]. One difficulty with this approach is that the derivative of $R g_{n}(R, R)$, used to express $V_{n}(R)$, has to be evaluated by a finite difference scheme, which is likely to give unreliable numerical results. It will be shown later (Theorem 3) that the second-order hyperbolic equation (2.22) can be replaced by an equivalent system of first-order coupled partial-differential equations that do not require the evaluation of the derivative of $R g_{n}(R, R)$. By using a finite difference discretization scheme with mesh size $\Delta=$ $R / N$ for these equations, it will be shown that only $O\left(N^{2}\right)$ operations are required to compute $g_{n}(R, \cdot)$, instead of $O\left(N^{3}\right)$ for methods that do not exploit the structure of $k_{n}$.

Alternately, we may choose to compute the normalized filter

$$
\bar{g}_{n}(R, r) \triangleq(R r)^{1 / 2} g_{n}(R, r),
$$

which is associated with the normalized estimation problem (2.18). In this case $\bar{g}_{n}$ satisfies the partial-differential equation

$$
\bar{\delta}_{n} \bar{g}_{n}(R, r)=V_{n}(R) \bar{g}_{n}(R, r),
$$

where

$$
\bar{\delta}_{n}=\left(\frac{\partial^{2}}{\partial R^{2}}-\frac{1}{R^{2}}\left(n^{2}-\frac{1}{4}\right)\right)-\left(\frac{\partial^{2}}{\partial r^{2}}-\frac{1}{r^{2}}\left(n^{2}-\frac{1}{4}\right)\right)
$$

is the normalized displacement operator, and where

$$
V_{n}(R)=-2 \frac{d}{d R} \bar{g}_{n}(R, R) .
$$

This formulation will turn out to be quite useful in Section $\mathrm{V}$, where our estimation results will be interpreted from an inverse spectral point of view.
The function $V_{n}(\cdot)$, which parametrizes (2.22) and (2.26), can be interpreted as follows. Let

$$
e_{n}^{2}(R)=E\left[\tilde{z}_{n}^{2}(R \mid R)\right]
$$

be the mean-square error for the filtering problem (2.9). By using the orthogonality property of linear least-squares estimates, we find that

$$
e_{n}^{2}(R)=E\left[z_{n}(R) \tilde{z}_{n}(R \mid R)\right]=\frac{1}{2 \pi} g_{n}(R, R),
$$

so that $V_{n}(\cdot)$ can be interpreted as

$$
V_{n}(R)=-4 \pi \frac{d}{d R}\left(R e_{n}^{2}(R)\right),
$$

the negative rates of change of the normalized mean-square error, $R e_{n}^{2}(R)$, as $R$ increases.

\section{Innovations Processes}

An interesting feature of the recursions associated with the partial-differential equation (2.22) is that in the process of computing $g_{n}(R, \cdot)$ they generate all the optimum filters $g_{n}(r, \cdot)$ for $0 \leq r \leq R$. These filters can be used to generate the filtered estimates $\hat{z}_{n}(r \mid r)$ and the innovations

$$
\nu_{n}(r)=y_{n}(r)-\hat{z}_{n}(r \mid r)
$$

for $0 \leq r \leq R$. Here $\nu_{n}(\cdot)$ is a white Gaussian noise process with intensity

$$
E\left[\nu_{n}(r) \nu_{n}(s)\right]=\frac{1}{2 \pi r} \delta(r-s) .
$$

Furthermore, if $V_{R}^{n}=H\left(\nu_{n}(r), 0 \leq r \leq R\right)$ denotes the Hilbert space spanned by $\nu_{n}(\cdot)$, we have

$$
V_{R}^{n}=Y_{R}^{n},
$$

and it is therefore equivalent to use $\nu_{n}(\cdot)$ or $y_{n}(\cdot)$ to solve the estimation problem (2.9).

\section{General Estimation Procedure}

In the previous section, we have seen that the problem of estimating a 2-D isotropic random field over a disk can be decomposed into a countable set of 1-D estimation problems. We will now describe the resulting estimation procedure. Let $\boldsymbol{a}$ be an arbitrary zero-mean random variable, whose joint statistics with $y(\cdot)$ are Gaussian and such that

$$
E\left[\mathbf{a} y_{n}^{*}(r)\right]=a_{n}(r) .
$$

A special case of interest is when $a=z(s)$, with $s \in D_{R}$, a sample of the random field that we observe. In this case estimation of $a$ is a smoothing problem, and the function $a_{n}(r)$ is $k_{n}(r, s) \exp (-j n \phi)$ where $(s, \phi)$ are the polar coordinates of $s$.

For the general case, the orthogonal decomposition (2.11b) shows that the conditional mean of $a$ given $Y_{R}$ can be expressed as

$$
E\left[a \mid Y_{R}\right]=\sum_{n=-\infty}^{\infty} E\left[a \mid Y_{R}^{n}\right]
$$


with

$$
E\left[\boldsymbol{a} \mid Y_{R}^{n}\right]=\int_{0}^{R} c_{n}(R, s) y_{n}(s) s d s,
$$

and by noting that the error $\tilde{\boldsymbol{a}}=\boldsymbol{a}-E\left[\boldsymbol{a} \mid Y_{R}\right]$ is orthogonal to $Y_{R}^{n}$ for all $n$, we find that

$$
a_{n}(r)=\int_{0}^{R} k_{n}(r, s) c_{n}(R, s) s d s+\frac{1}{2 \pi} c_{\pi}(R, r)
$$

for $0 \leq r \leq R$.

To guarantee the existence and unicity of a solution to (3.3), we assume as above that $k(\cdot) \in L_{2}(r d r ;[0, R])$, so the operator $I / 2 \pi+K_{n}$ is invertible over $L_{2}(r d r ;[0, R])$. Then, to compute $c_{n}(R, \cdot)$, one method would be to discretize the interval $[0, R]$ into $N$ subintervals of length $\Delta=R / N$ and to solve the resulting system of linear equations. However, this methods requires $O\left(N^{3}\right)$ operations and a more efficient approach is to use the following result

Theorem 2: If $k$ and $a_{n} \in L_{2}(r d r ;[0, R])$, the filter $c_{n}(R, \cdot)$ obeys the equation

$$
\frac{\partial}{\partial R} c_{n}(R, r)=-R c_{n}(R, R) g_{n}(R, r)
$$

for $0 \leq r \leq R$, where $c_{n}(R, R)$ is evaluated by setting $r=R$ in (3.3).

Proof: Take the partial derivative of (3.3) with respect to $R$. This gives

$$
\begin{aligned}
-R c_{n}(R, R) k_{n}(r, R)=\int_{0}^{R} k_{n}(r, s) & \frac{\partial}{\partial R} c_{n}(r, s) s d s \\
& +\frac{1}{2 \pi} \frac{\partial}{\partial R} c_{n}(R, r),
\end{aligned}
$$

so that, by linearity, if we compare this equation to (2.21) and use the unicity of the solution to (2.21), we obtain (3.4).

To compute $c_{n}(\cdot, \cdot)$ one needs only to propagate the recursions (3.4) simultaneously with (2.22) for $g_{n}(\cdot, \cdot)$, starting from $R=0$. It will be shown in Section IV that by discretizing these equations with the step size $\Delta=R / N$, one gets a numerical scheme that requires only $O\left(N^{2}\right)$ operations to compute $c_{n}(R, \cdot)$, so that this method is very efficient.

The estimation procedure described above requires the computation of the filters $c_{n}$ and $g_{n}$ for all integers $n$. However, in Section V it will be shown that the functions $\gamma_{n}$ that are obtained by taking the Hankel transform of order $n$ of $\delta(r-s)-g_{n}(r, s)$ with respect to $s$ can be computed easily from one another. In addition, there exist several cases that require considerably less work.

Example: Assume that we want to estimate the random field $z(\cdot)$ at the origin of the disk $D_{R^{\prime}}$, i.e., $a=z(0)$. In this case we have $z(0)=z_{0}(0)$, so that $z(0)$ is uncorrelated with the processes $y_{n}$ for $n \neq 0$, and the estimate

$$
E\left[z(\mathbf{0}) \mid Y_{R}\right]=\int_{0}^{R} c_{0}(R, s) y_{0}(s) s d s
$$

depends only on the filter $c_{0}(R, \cdot)$, so that only $c_{0}$ and $g_{0}$ need to be computed.

The problem of estimating the random field $z(\cdot)$ at the origin of the disk $D_{R}$ might arise when noisy observations of $z(\cdot)$ are given over a large domain, and we want to construct a smoothed estimate of the field over this domain. In this case a simple smoothing procedure is to base the estimate at a given point $r$ on the observations over a disk $D_{R}(\boldsymbol{r})$ centered at this point. The recursions obtained above for $c_{0}(R, \cdot)$ and $g_{0}(R, \cdot)$ show that this smoothing procedure requires the same amount of computation as the Levinson recursions of 1-D linear prediction [1], [2], which is somewhat surprising in light of the fact that here we are solving a 2-D estimation problem, instead of a 1-D one.

\section{FIRST-ORDER EQUATIONS}

As was noted above, the second-order equation (2.22) for $g_{n}$ can be replaced by a system of coupled first-order equations for $g_{n}$ and $g_{n+1}$, which are easier to propagate. As we will see below, these equations have a structure very similar to that of the continuous-time Levinson recursions. The key step is to observe that the covariance kernels $k_{n}$ have the following property.

Lemma 2: If $k(\cdot)$ is differentiable, we have

$$
\left(\frac{\partial}{\partial r}-\frac{n}{r}\right) k_{n}(r, s)+\left(\frac{\partial}{\partial s}+\frac{(n+1)}{s}\right) k_{n+1}(r, s) \equiv 0
$$

$$
\left(\frac{\partial}{\partial s}-\frac{n}{s}\right) k_{n}(r, s)+\left(\frac{\partial}{\partial r}+\frac{(n+1)}{r}\right) k_{n+1}(r, s) \equiv 0
$$

for all $n$.

Proof: The kernel $k_{n}(\cdot, \cdot)$ can be expressed as (2.13), and the Bessel functions $\left\{J_{n}(\cdot), n \in \mathbb{Z}\right\}$ obey the recursions

$$
\begin{aligned}
& \left(\frac{d}{d r}+\frac{n}{r}\right) J_{n}(\lambda r)=\lambda J_{n-1}(\lambda r) \\
& \left(\frac{d}{d r}-\frac{n}{r}\right) J_{n}(\lambda r)=-\lambda J_{n+1}(\lambda r) .
\end{aligned}
$$

This implies that

$$
\begin{aligned}
\left(\frac{\partial}{\partial r}-\frac{n}{r}\right) k_{n}(r, s) & =-\int_{0}^{\infty} J_{n+1}(\lambda r) J_{n}(\lambda s) \hat{k}(\lambda) \lambda d \lambda \\
& =-\left(\frac{\partial}{\partial s}+\frac{(n+1)}{s}\right) k_{n+1}(r, s),
\end{aligned}
$$

so that (4.1a) is satisfied. The relation (4.16) can be obtained by symmetry.

The displacement property (2.15) of the covariance kernel $k_{n}(\cdot, \cdot)$ is a direct consequence of Lemma 2. To see this, 
note that the displacement operator $\boldsymbol{\delta}_{n}$ can be expressed as $\boldsymbol{\delta}_{n}=\mathbf{B}_{n}(r)-\mathbf{B}_{n}(s)$, where

$$
\begin{aligned}
\mathbf{B}_{n}(r) & \triangleq\left(\frac{d}{d r}+\frac{(n+1)}{r}\right)\left(\frac{d}{d r}-\frac{n}{r}\right) \\
& =\frac{d^{2}}{d r^{2}}+\frac{1}{r} \frac{d}{d r}-\frac{n^{2}}{r^{2}},
\end{aligned}
$$

so that by combining (4.1a) and (4.1b), we obtain (2.15). We can also use Lemma 2 to establish the following result.

Theorem 3: If $k(\cdot)$ is differentiable, the filters $g_{n}$ and $g_{n+1}$ satisfy the system of first-order equations

$$
\begin{array}{r}
\left(\frac{\partial}{\partial R}-\frac{n}{R}\right) g_{n}(R, r)+\left(\frac{\partial}{\partial r}+\frac{(n+1)}{r}\right) g_{n+1}(R, r) \\
=-\rho_{n}(R) g_{n}(R, r) \\
\left(\frac{\partial}{\partial r}-\frac{n}{r}\right) g_{n}(R, r)+\left(\frac{\partial}{\partial R}+\frac{(n+1)}{R}\right) g_{n+1}(R, r) \\
=\rho_{n}(R) g_{n+1}(R, r),
\end{array}
$$

where

$$
\rho_{n}(R) \triangleq R\left(g_{n}(R, R)-g_{n+1}(R, R)\right) .
$$

Proof: Operate respectively with $(\partial / \partial R)-(n / R)$ and $(\partial / \partial r)+((n+1) / r)$ on the integral equations satisfied by $g_{n}$ and $g_{n+1}$ and add the resulting equations. Using the displacement property (4.1a) of $k_{n}$ and $k_{n+1}$, and integrating by parts gives

$0=\rho_{n}(R) k_{n}(r, R)+\int_{0}^{R} k_{n}(r, s) m(R, s) s d s$

$$
+\frac{1}{2 \pi} m(R, r)
$$

where

$$
\begin{aligned}
m(R, r) \triangleq\left(\frac{\partial}{\partial R}-\frac{n}{R}\right) g_{n}(R, r) & \\
& +\left(\frac{\partial}{\partial r}+\frac{(n+1)}{r}\right) g_{n+1}(R, r)
\end{aligned}
$$

and where $\rho_{n}(\cdot)$ satisfies (4.4). This equation can be viewed as obtained from $(2.21)$ by multiplication by $-\rho_{n}(R)$. Therefore, by linearity, and since the solution of (2.21) is unique, we have

$$
m(R, r)=-\rho_{n}(R) g_{n}(R, r) .
$$

The identity ( $4.3 \mathrm{~b}$ ) can be derived similarly.

The structure of the first-order equations (4.3) is reminiscent of the continuous-time Levinson recursions [7], [11]. As expected, these equations are equivalent to the second-order equation (2.22). Indeed, by combining (4.3a) and (4.3b), we obtain (2.22) with

$$
\begin{gathered}
V_{n}(R)=\rho_{n}^{2}(R)-\dot{\rho}_{n}(R)-\frac{(2 n+1)}{R} \rho_{n}(R) \\
V_{n+1}(R)=\rho_{n}^{2}(R)+\dot{\rho}_{n}(R)-\frac{(2 n+1)}{R} \rho_{n}(R) .
\end{gathered}
$$

The relation (4.5) shows that the potential functions $V_{n}(\cdot)$ and $V_{n+1}(\cdot)$ are entirely specified by $\rho_{n}(\cdot)$, which plays a role similar to that of the reflectivity function of the Levinson recursions. Conversely, given either $V_{n}(\cdot)$ or $V_{n+1}(\cdot)$ with $n \geq 0$, to compute $\rho_{n}(\cdot)$ we need only to solve the Riccati equation (4.5a) or (4.5b) with the initial condition

$$
\begin{aligned}
\lim _{R \rightarrow 0} R^{-(2 n+1)} \rho_{n}(R) & =\lim _{R \rightarrow 0} R^{-2 n} g_{n}(R, R) \\
& =\frac{2 \pi}{\left(2^{n} n !\right)^{2}} \int_{0}^{\infty} \lambda^{2 n+1} \hat{k}(\lambda) d \lambda,
\end{aligned}
$$

which is obtained by setting $r=R$ and letting $R \rightarrow 0$ in the integral equation (2.21).

\section{A. Discretization Scheme}

To compute $g_{n}(R, \cdot)$ and $c_{n}(R, \cdot)$, we can divide the interval $[0, R]$ into $N$ subintervals of length $\Delta=R / N$ and discretize (4.3) and (3.4) accordingly. If $G_{n}(k, l) \triangleq$ $g_{n}(k \Delta, l \Delta)$ and $C_{n}(k, l) \triangleq c_{n}(k \Delta, l \Delta)$, and if at stage $K$ we assume that $G_{n}(K, l), G_{n+1}(K, l)$, and $C_{n}(K, l)$ have been computed for $0 \leq l \leq K$, the recursions that we obtain are

$$
\begin{aligned}
G_{n}(K+1, l)= & \left(1+\frac{n}{K}-R_{n}(K) \Delta\right) G_{n}(K, l) \\
& +G_{n+1}(K, l-1) \\
& -\left(1+\frac{n+1}{l}\right) G_{n+1}(K, l) \\
G_{n+1}(K+1, l)= & \left(1-\frac{n+1}{K}+R_{n}(K) \Delta\right) G_{n+1}(K, l) \\
& +G_{n}(K, l-1)-\left(1-\frac{n}{l}\right) G_{n}(K, l),
\end{aligned}
$$

with $R_{n}(K)=K\left(G_{n}(K, K)-G_{n+1}(K, K)\right) \Delta$ and $1 \leq l \leq$ $K$, and

$$
C_{n}(K+1, l)=C_{n}(K, l)-K C_{n}(K, K) G_{n}(K, l) \Delta^{2}
$$

for $0 \leq l \leq K$. The boundary condition (2.25) gives

$$
\begin{aligned}
& G_{0}(K+1,0)=G_{0}(K+1,1) \\
& G_{n}(K+1,0)=0, \quad \text { for } n \neq 0,
\end{aligned}
$$

and by discretizing the integral equations (2.21) and (3.3), we find that

$$
\begin{array}{r}
\frac{1}{2 \pi} G_{n}(K+1, K+1)=k_{n}((K+1) \Delta,(K+1) \Delta) \\
-\sum_{l=0}^{K} k_{n}((K+1) \Delta, l \Delta) G_{n}(K+1, l) l \Delta^{2}
\end{array}
$$

and

$$
\begin{aligned}
\frac{1}{2 \pi} C_{n}(K & +1, K+1)=a_{n}((K+1) \Delta) \\
& -\sum_{l=0}^{K} k_{n}((K+1) \Delta, l \Delta) C_{n}(K+1, l) l \Delta^{2} .
\end{aligned}
$$


The functions $G_{n}(K, \cdot), G_{n+1}(K, \cdot)$, and $C_{n}(K, \cdot)$ can therefore be computed recursively, starting from $K=0$. The number of operations required at each step is $8 K$, so that the total number of operations required to compute $G_{n}(N, \cdot), G_{n+1}(N, \cdot)$, and $C_{n}(N, \cdot)$ is $4 N^{2}$. The amount of storage required is $3 N$.

The recursions (4.7)-(4.11) for $G_{n}(\cdot, \cdot), G_{n+1}(\cdot, \cdot)$, and $C_{n}(\cdot, \cdot)$ correspond to a simple finite-difference discretization of the coupled hyperbolic equations (4.3). To keep these recursions as simple as possible, we have used forward differences to approximate $\partial / \partial R^{\prime}$ and backward differences for $\partial / \partial r$. However, other choices are possible (see [12, Chapter 6]), such as a central-central (CC) scheme. Several factors intervene in the selection of a particular difference scheme: the most important is its stability, but another is accuracy, i.e., the magnitude of the truncation error. For the scheme described above the error is proportional to $\Delta$, whereas for a CC scheme it would be proportional to $\Delta^{2}$. Another important consideration is that, in order to take full advantage of the causality of equations (4.3) for $g_{n}$ and $g_{n+1}$, an explicit difference scheme should be selected, since implicit schemes are more costly from a computational point of view [12]. No matter what particular scheme is selected to discretize (4.3), the resulting recursions will be considerably more efficient than nonrecursive methods based on the results of Popov [3] and Yadrenko [4], [5, Chapter 4], since such methods would require $O\left(N^{3}\right)$ operations to compute $G_{n}(\cdot, \cdot)$ and $C_{n}(\cdot, \cdot)$.

Finally, for large values of $R$, instead of discretizing the first-order system (4.3), it may be preferable to discretize an equivalent system of equations for the normalized filters $\bar{g}_{n}(\cdot, \cdot)$ and $\bar{g}_{n+1}(\cdot, \cdot)$, since as $k \rightarrow \infty$, the normalized filtering problem (2.18) remains regular, whereas the unnormalized problem (2.9) becomes singular because the variance of the noise $v_{n}(\cdot)$ is $1 / 2 \pi r$.

\section{Spectral Domain Viewpoint}

As in the 1-D case, where the Levinson recursions can be expressed in the Fourier domain as equivalent recursions for the Krein polynomials [6], [7], the second-order and coupled first-order equations that we have obtained above for the estimation filters $g_{n}(\cdot, \cdot)$ have counterparts in the spectral domain. To obtain these recursions, we will use the isometry

$$
y(r) \leftrightarrow \exp (j \lambda \cdot \mathbf{r})
$$

between $Y$ and $L_{2}(\hat{r}(\lambda) \lambda d \lambda d \phi / 2 \pi)$, where $\lambda \cdot \mathbf{r}$ denotes the inner product of $\lambda$ and $r \in \mathbb{R}^{2},(\lambda, \phi)$ are the polar coordinates of $\lambda$, and

$$
\begin{aligned}
\hat{r}(\lambda) & =\frac{1}{2 \pi}+\hat{k}(\lambda) \\
& =\int_{0}^{\infty} J_{0}(\lambda l)\left(\frac{\delta(l)}{\pi l}+k(l)\right) l d l
\end{aligned}
$$

is the spectral density function of the field $y(\cdot)$. To check that (5.1) constitutes an isometry, note that

$$
\begin{aligned}
& E[y(\boldsymbol{r}) y(s)] \\
& \quad=\frac{\delta(l)}{\pi l}+k(l) \\
& \quad=\int_{0}^{\infty} J_{0}(\lambda l) \hat{r}(\lambda) \lambda d \lambda \\
& \quad=\int_{0}^{2 \pi} \int_{0}^{\infty} \exp (j \lambda \cdot(\boldsymbol{r}-\boldsymbol{s})) \frac{\hat{r}(\lambda)}{2 \pi} \lambda d \lambda d \phi \\
& =\langle\exp (j \lambda \cdot \boldsymbol{r}), \exp (j \lambda \cdot s)\rangle_{L_{2}(\hat{r}(\lambda) \lambda d \lambda d \phi / 2 \pi)}
\end{aligned}
$$

where $l=|\boldsymbol{r}-\boldsymbol{s}|$ and where we have used the property

$$
\frac{1}{2 \pi} \int_{0}^{2 \pi} \exp (j \lambda l \cos \theta) d \theta=J_{0}(\lambda l)
$$

of the Bessel function of order zero.

Note that, strictly speaking, $Y(\boldsymbol{r})$ and $\exp (j \lambda \cdot \boldsymbol{r})$ do not belong to $Y$ and $L_{2}(\hat{\mathrm{r}}(\lambda) \lambda d \lambda d \phi / 2 \pi)$, respectively, since $Y$ was defined in (2.6) as the Hilbert space obtained by integrating $y(\cdot)$ with respect to square-integrable functions. Thus, the map (5.1) should be interpreted as

$$
\int b(\boldsymbol{r}) y(\boldsymbol{r}) d r \leftrightarrow \int b(\boldsymbol{r}) \exp (j \lambda \cdot \boldsymbol{r}) d r,
$$

where $b \in L_{2}\left(\mathbb{R}^{2}\right)$ is arbitrary. However, for convenience, we will continue to use $y(\boldsymbol{r})$ and $\exp j \lambda \cdot \boldsymbol{r}$ as the defining elements of the isometry (5.1).

Under this isometry, the Fourier coefficient process $y_{n}(r)$ is mapped into

$$
\frac{1}{2 \pi} \int_{0}^{2 \pi} \exp j(\lambda \cdot r-n \theta) d \theta=j^{n} J_{n}(\lambda r) \exp (-j n \phi),
$$

where $(r, \theta)$ are the polar coordinates of $r$ and where we have used the Fourier series expansion

$$
\exp j \lambda \cdot \boldsymbol{r}=\sum_{n=-\infty}^{\infty} j^{n} J_{n}(\lambda r) \exp (j n(\theta-\phi)) .
$$

The orthogonality of the Fourier coefficient processes $y_{n}(\cdot)$ and $y_{m}(\cdot)$ for $n \neq m$ is reflected by the fact that

$$
E\left[y_{n}(r) y_{m}^{*}(s)\right]=\int_{0}^{\infty} J_{n}(\lambda r) J_{n}(\lambda s) \hat{r}(\lambda) \lambda d \lambda \delta_{n m},
$$

with

$$
\begin{aligned}
\delta_{n m} & =\frac{1}{2 \pi} \int_{0}^{2 \pi} \exp (j(m-n) \phi) d \phi \\
& =1 \text { for } n=m, \quad 0 \text { otherwise, }
\end{aligned}
$$

so that the orthogonal decomposition (2.11b) of the observation space corresponds to the decomposition

$$
S_{R}=\bigoplus_{n=-\infty}^{\infty} S_{R}^{n}
$$

of the subspace $S_{R}$ of $L_{2}(\hat{r}(\lambda) \lambda d \lambda d \phi / 2 \pi)$, which is spanned by $\{\exp j \lambda \cdot r, 0 \leq r \leq R\}$ and where $S_{R}^{n}$ is the subspace spanned by $\left\{J_{n}(\lambda r) \exp -j n \phi, 0 \leq r \leq R\right\}$. 


\section{A. Schrödinger Equations}

The identity (5.8) shows that within the larger isometry between $Y$ and $L_{2}(\hat{r}(\lambda) \lambda d \lambda d \phi / 2 \pi)$, we can construct a smaller isometry between $Y^{n}$ and $L_{2}(\hat{r}(\lambda) \lambda d \lambda)$ that is defined by

$$
y_{n}(r) \leftrightarrow J_{n}(\lambda r) .
$$

In this framework the Fourier coefficient estimation problem (2.9) is equivalent to the one of orthogonalizing the functions $\left\{J_{n}(\lambda s), 0 \leq s \leq r\right\}$, and the innovations process $\nu_{n}(r)$ is mapped into

$$
\gamma_{n}(r, \lambda) \triangleq J_{n}(\lambda r)-\int_{0}^{r} g_{n}(r, s) J_{n}(\lambda s) s d s .
$$

The functions $\gamma_{n}(r, \cdot)$ can be viewed as Hankel transforms of order $n$ of $\delta(r-s)-g_{n}(r, s)$ with respect to $s$. They play the same role as the Krein polynomials [6], [7] in one dimension. Since the innovations process $\nu_{n}(r)$ is white with intensity $1 / 2 \pi r$, the functions $\gamma_{n}(r, \lambda)$ are orthogonal and

$$
\int_{0}^{\infty} \gamma_{n}(r, \lambda) \gamma_{n}(s, \lambda) \hat{r}(\lambda) \lambda d \lambda=\frac{1}{2 \pi r} \delta(r-s) .
$$

To characterize $\gamma_{n}(\cdot, \cdot)$, instead of using the function $g_{n}(\cdot, \cdot)$ as in $(5.11)$, we will rely on the following result.

Theorem 4: For $n \geq 0$ the function $\gamma_{n}(r, \lambda)$ satisfies the differential equation

$$
\left(\frac{d^{2}}{d r^{2}}+\frac{1}{r} \frac{d}{d r}+\left(\lambda^{2}-V_{n}(r)-\frac{n^{2}}{r^{2}}\right)\right) \gamma_{n}(r, \lambda)=0
$$

with the initial condition

$$
\lim _{r \rightarrow 0} 2^{n} n !(\lambda r)^{-n} \gamma_{n}(r, \lambda)=1,
$$

and for $n \leq 0$ we have

$$
\gamma_{n}(r, \lambda)=(-1)^{n} \gamma_{-n}(r, \lambda)
$$

Proof: Operate with $\boldsymbol{B}_{n}(r)$ (defined in (4.2)) on (5.11). This gives

$$
\begin{aligned}
\boldsymbol{B}_{n}(r) \gamma_{n}(r, \lambda)= & -\left(\lambda^{2}-V_{n}(r)\right) J_{n}(\lambda r) \\
& +\left.r \frac{\partial}{\partial s} g_{n}(r, s)\right|_{s=r} J_{n}(\lambda r) \\
& -\lambda r g_{n}(r, r) \frac{d}{d r} J_{n}(\lambda r) \\
& -\int_{0}^{r}\left(\boldsymbol{B}_{n}(r) g_{n}(r, s)\right) J_{n}(\lambda s) s d s .
\end{aligned}
$$

Then, since the displacement operator can be expressed as $\boldsymbol{\delta}_{n}=\boldsymbol{B}_{n}(\mathrm{r})-\boldsymbol{B}_{n}(s)$, by using (2.22) for $g_{n}(\cdot, \cdot)$ and integrating by parts, we obtain (5.13). The initial condition (5.14) is equivalent to the one satisfied by $J_{n}(\lambda r)$ and can be derived from (5.11). Note that only one initial condition is needed to specify the solution of (5.13), since only one solution of (5.13) is regular at $r=0$.

Equation (5.13) is a perturbed Bessel equation. To use it to compute the functions $\gamma_{n}(r, \cdot)$ for increasing values of $r$, we need to find an expression for the potential $V_{n}(r)$ appearing in this equation. To do so we use the definition (2.27) of $V_{n}(r)$ and note from (2.28) that

$$
\begin{aligned}
g_{n}(r, r) & =2 \pi E\left[\tilde{z}_{n}^{2}(r \mid r)\right] \\
& =2 \pi \int_{0}^{\infty} \gamma_{n}(r, \lambda) J_{n}(\lambda r) \hat{k}(\lambda) \lambda d \lambda,
\end{aligned}
$$

where the second identity is obtained by observing that there exists an isometry $z_{n}(r) \leftrightarrow J_{n}(\lambda r)$ between $Z^{n}$ and $L_{2}(\hat{k}(\lambda) \lambda d \lambda)$ that is similar to the isometry (5.10) between $Y^{n}$ and $L_{2}(\hat{r}(\lambda) \lambda d \lambda)$. The potential $V_{n}(r)$ can therefore be expressed as

$$
V_{n}(r)=-4 \pi \frac{d}{d r}\left(r \int_{0}^{\infty} \gamma_{n}^{2}(r, \lambda) J_{n}(\lambda r) \hat{k}(\lambda) \lambda d \lambda\right)
$$

and by substituting this relation inside (5.13), we obtain a nonlinear second-order equation that can be used to compute $\gamma_{n}(r, \lambda)$ recursively. This equation is the exact analogue of the recursions satisfied by the Krein polynomials in one dimension.

In terms of the normalized function

$$
\phi_{n}(r, \lambda) \triangleq(r \lambda)^{1 / 2} \gamma_{n}(r, \lambda)
$$

(5.13) becomes

$$
\left(\frac{d^{2}}{d r^{2}}+\left(\lambda^{2}-V_{n}(r)-\frac{1}{r^{2}}\left(n^{2}-\frac{1}{4}\right)\right)\right) \phi_{n}(r, \lambda)=0
$$

with

$$
\lim _{r \rightarrow 0} 2^{n} n !(\lambda r)^{-(n+(1 / 2))} \phi_{n}(r, \lambda)=1,
$$

which is the Schrödinger equation satisfied by a particle with angular momentum $n$ and energy $E=\lambda^{2}$ moving inside a circulary symmetric potential well in a space of dimension two; the expression (5.16) for the potential $V_{n}(\cdot)$ becomes

$$
V_{n}(r)=-4 \pi \frac{d}{d r}\left(\int_{0}^{\infty} \phi_{n}(r, \lambda) u_{n}(\lambda r) \hat{k}(\lambda) d \lambda\right),
$$

where

$$
u_{n}(\lambda r) \triangleq(r \lambda)^{1 / 2} J_{n}(\lambda r) .
$$

The relations (5.18)-(5.20) will be used below to obtain an inverse spectral interpretation of our estimation procedure. 


\section{B. Transformation Operators}

The first-order equations (4.3) for $g_{n}$ and $g_{n+1}$ can also be transformed to the spectral domain. This gives

$$
\begin{gathered}
\frac{d}{d r} \gamma_{n}(r, \lambda)=\left(\frac{n}{r}-\rho_{n}(r)\right) \gamma_{n}(r, \lambda)-\lambda \gamma_{n+1}(r, \lambda) \\
\frac{d}{d r} \gamma_{n+1}(r, \lambda)=\lambda \gamma_{n}(r, \lambda)-\left(\frac{n+1}{r}-\rho_{n}(r)\right) \gamma_{n+1}(r, \lambda),
\end{gathered}
$$

and by combining the relations (4.4) and (5.15), we obtain

$$
\begin{aligned}
\rho_{n}(r)=2 \pi r \int_{0}^{\infty} & \left(\gamma_{n}(r, \lambda) J_{n}(\lambda r)\right. \\
& \left.-\gamma_{n+1}(r, \lambda) J_{n+1}(\lambda r)\right) \hat{k}(\lambda) \lambda d \lambda .
\end{aligned}
$$

When (5.22) is substituted inside (5.21), we get a set of nonlinear first-order equations for $\gamma_{n}$ and $\gamma_{n+1}$ which have almost the same form as the 1-D Krein recursions [6], [7]. When discretized, these equations can be used to compute $\gamma_{n}(r, \cdot)$ for increasing values of $r$, and the resulting numerical scheme has the same complexity as the method described in Section IV for computing $g_{n}$ and $g_{n+1}$.

An additional feature of the first-order equations (5.21) is that they may be used to show that the functions $\gamma_{n}(\cdot, \cdot)$ can all be generated from one another. This property is very important, since we have seen in Section III that to solve the 2-D estimation problem (2.1) in full generality, i.e., to estimate the random field $z(r)$ at a point other than the origin of the observation disk, the functions $g_{n}(\cdot, \cdot)$ or $\gamma_{n}(\cdot, \cdot)$ must be computed for all integers $n$.

Let $w_{n}(\cdot)$ and $u_{n}(\cdot)$ be given by

$$
\begin{aligned}
\rho_{n}(r) & =\frac{n}{r}-\frac{\dot{w}_{n}(r)}{w_{n}(r)} \\
& =\frac{(n+1)}{r}+\frac{\dot{u}_{n}(r)}{u_{n}(r)}
\end{aligned}
$$

with the initial conditions

$$
\begin{gathered}
\lim _{r \rightarrow 0} r^{-n} w_{n}(r)=1 \\
\lim _{r \rightarrow 0} r^{n+1} u_{n}(r)=1
\end{gathered}
$$

Then, the first-order equations (5.21) can be expressed as

$$
\begin{gathered}
\lambda \gamma_{n+1}(r, \lambda)=-W\left[\gamma_{n}(r, \lambda), w_{n}(r)\right] / w_{n}(r) \\
\lambda \gamma_{n}(r, \lambda)=W\left[\gamma_{n+1}(r, \lambda), u_{n}(r)\right] / u_{n}(r),
\end{gathered}
$$

where

$$
W[f(r), g(r)] \triangleq \dot{f}(r) g(r)-f(r) \dot{g}(r)
$$

denotes the Wronskian of $f(\cdot)$ and $g(\cdot)$. By substituting (5.23a) and (5.23b) inside the Riccati equations (4.5a) and (4.5b), respectively, we obtain

$$
\begin{array}{r}
\left(\frac{d^{2}}{d r^{2}}+\frac{1}{r} \frac{d}{d r}-\left(V_{n}(r)+\frac{n^{2}}{r^{2}}\right)\right) w_{n}(r)=0 \\
\left(\frac{d^{2}}{d r^{2}}+\frac{1}{r} \frac{d}{d r}-\left(V_{n+2}(r)+\frac{(n+1)^{2}}{r^{2}}\right)\right) u_{n}(r)=0,
\end{array}
$$

so that $w_{n}(\cdot)$ and $u_{n-1}(\cdot)$ are two solutions of the equation obtained by setting $\lambda=0$ in the differential equation (5.13). However, $w_{n}$ is regular at the origin, whereas $u_{n-1}$ has a singularity of order $n$ at $r=0$. So the relations (5.24) show that by operating on $\gamma_{n}$ with the regular solution $w_{n}$, we generate $\gamma_{n+1}$, whereas by operating on it with the singular solution $u_{n-1}$, we generate $\gamma_{n-1}$.

To be able to use the recursion (5.24a) to generate all $\gamma_{n}(\cdot, \cdot)$ for $n \geq 0$ from $\gamma_{0}(\cdot, \cdot)$, we must show that the solution $w_{n+1}$ can be computed from $w_{n}$. 'lo do so note that

$$
w_{n}(r)=\lim _{\lambda \rightarrow 0} 2^{n} n ! \gamma_{n}(r, \lambda) / \lambda^{n}
$$

so that by taking limits inside (5.24a) and noting that

$$
W\left[\gamma_{n}(r, \lambda), w_{n}(r)\right]=-\frac{\lambda^{2}}{r} \int_{0}^{r} \gamma_{n}(s, \lambda) w_{n}(s) s d s,
$$

we find that

$$
w_{n+1}(r)=\frac{1}{r w_{n}(r)} \int_{0}^{r} w_{n}^{2}(s) s d s .
$$

Similarly, by subtracting (5.23a) from (5.23b) and integrating, one gets

$$
u_{n}(r)=1 /\left(r w_{n}(r)\right)
$$

so that the singular solution $u_{n}$ can also be generated from $w_{n}$.

The transformations (5.24) were introduced by Crum [13] and were later used by Krein [14] and Agranovich and Marchenko [15] to study the inverse scattering problem of quantum mechanics for waves of nonzero angular momentum. The main property of these transformations is that they are isospectral, i.e., they transform the solutions of the Schrödinger equation associated with the potential $V_{n}(\cdot)$ and angular momentum $n$ into solutions of the equation associated with the potential

$$
\begin{aligned}
V_{n+1}(r) & =V_{n}(r)+2 \dot{\rho}_{n}(r) \\
& =V_{n}(r)-2 \frac{d^{2}}{d r^{2}} \ln \left(w_{n}(r) / r^{n}\right)
\end{aligned}
$$

and angular momentum $n+1$, and vice versa, but they keep invariant the spectral density function $\hat{r}(\lambda)$ of

$$
\Delta_{n}=-\frac{d^{2}}{d r^{2}}+\left(V_{n}(r)-\frac{1}{r^{2}}\left(n^{2}-\frac{1}{4}\right)\right)
$$

and $\Delta_{n+1}$. To see this, note from (5.12) that $\hat{r}(\lambda)$ is the spectral density of all Schrödinger operators $\Delta_{n}$. 


\section{Inverse Spectral Interpretation}

The results of this paper have an inverse spectral interpretation. Indeed, as was noted above, a consequence of our estimation procedure is that we generate a sequence of Schrödinger operators $\Delta_{n}, n \in \mathbb{N}$ whose spectral density $\hat{r}(\lambda)$ is the same as that of the random field $y(\cdot)$. The operators $\Delta_{n}$ are entirely characterized by the potentials $V_{n}(\cdot)$, which unfortunately are unknown. However, the recursions (5.18)-(5.20) for $\phi_{n}(r, \lambda)$ can be viewed as reconstructing $V_{n}(r)$ recursively from $\hat{k}(\lambda)=\hat{r}(\lambda)-1 / 2 \pi$. The identity (5.20) for $V_{n}(r)$ is similar to the trace formula used by Deift and Trubowitz [16] for solving the inverse scattering problem on the real line.

The expression (2.23) for $V_{n}(r)$, which was derived in Section II, has also an inverse spectral interpretation. Given $\hat{r}(\lambda)$, a procedure proposed by Gelfand and Levitan [17] to reconstruct the potential $V_{n}(r)$ associated with $\hat{r}(\lambda)$ is as follows. Let

$$
\bar{k}_{n}(r, s)=(r s)^{1 / 2} \int_{0}^{\infty} J_{n}(\lambda r) J_{n}(\lambda s)\left(\hat{r}(\lambda)-\frac{1}{2 \pi}\right) \lambda d \lambda .
$$

Then, solve the integral equation

$$
\bar{k}_{n}(r, R)=\int_{0}^{k} \bar{k}_{n}(r, s) \bar{g}_{n}(R, s) d s+\frac{1}{2 \pi} \bar{g}_{n}(R, r)
$$

for $0 \leq \mathrm{r} \leq \mathrm{R}$. The potential $V_{n}(\cdot)$ is given by

$$
V_{n}(R)=-2 \frac{d}{d R} \bar{g}_{n}(R, R) \text {. }
$$

This procedure is identical to the estimation method described in Section II.

However, there is an important difference between the random field estimation and inverse spectral problems. The primary objective of the estimation problem is to compute the functions $g_{n}(r, \cdot)$ or $\gamma_{n}(r, \cdot)$, whereas for the inverse spectral problem we are only interested in the potential $V_{n}(\cdot)$. In the inverse spectral context the triangular kernel $\bar{g}_{n}(\cdot, \cdot)$ relates the solutions $\phi_{n}(r, \lambda)$ of the Schrödinger equation (5.18) to the free solutions, i.e., to the solutions obtained by setting $V_{n}(r) \equiv 0[18, \mathrm{p} .45]$.

\section{CONCLUUSION}

In this paper we have obtained an efficient method for constructing the estimates of a 2-D isotropic random field given noisy observations of this field over a finite disk. This method exploits the covariance structure of the field to obtain some recursions for the optimum filter as the radius of the observation disk increases. The resulting algorithm is very efficient and generalizes the Levinson recursions [1], [2] of 1-D linear prediction. An inverse spectral interpretation of this estimation procedure was also given.

The analysis developed in this paper can be extended easily to isotropic random fields in higher dimensions, provided that instead of expanding the random field in
Fourier series, we expand it in spherical harmonics as in [5, p. 5] and [20, p. 254]. However, the results described here depend heavily on the assumption that the observed field is isotropic. In the inverse spectral context this assumption implies that the potentials that we reconstruct are circularly symmetric. When the observed field is not isotropic, but only homogeneous, it is not clear whether results in multidimensional inverse spectral theory will be useful to solve the random fields estimation problem. Another possible research direction would be to see whether the ideas discussed in this paper can be used to study the various extrapolation and interpolation problems for isotropic random fields discussed in Chapter 4 of the recent monograph of Yadrenko [5].

\section{REFERENCES}

[1] N. Levinson, "The Wiener rms (root mean square) error criterion in filter design and prediction," J. Math. Phys., vol. 25, pp. 261-278, Jan. 1947.

[2] T. Kailath, "A view of three decades of linear filtering theory," IEEE Trans. Inform. Theory, vol. IT-20, no. 2, pp. 146-181, Mar. 1974.

[3] Yu. D. Popov, "On linear extrapolation of a homogeneous and isotropic random field by discrete observations on a circle," 'Theory Probab. Math. Statist., no. 4, pp. 115-120, 1974.

[4] M. I. Yadrenko, "On a linear extrapolation problem for an isotropic random field," Theory Probab. Math. Statist., no. 1, pp. 239-247, 1974.

[5] , Spectral Theory of Random Fields. New York: Optimization Software, 1983.

[6] M. G. Krein, "The continuous analogues of theorems on polynomials orthogonal on the unit circle," Dokl. Akad. Nauk SSSR, vol. 104, pp. 637-640, 1955.

[7] T. Kailath, A. Vieira, and M. Morf, "Inverses of Toeplitz operators, innovations, and orthogonal polynomials," SIAM Rev., vol. 20, no. 1, pp. 106-119, Jan. 1978.

[8] A. M. Yaglom, "Second order homogeneous random fields," in Proc. 4th Berkeley Symp. Math. Statist. and Probab., vol. 2, 1961, pp. 593-622.

[9] E. Wong, "Two-dimensional random fields and representation of images," SIAM J. Applied Math., vol. 16, no. 4, pp. 756-770, July 1968.

[10] R. J. Adler, The Geometry of Random Fields. Chichester, England: Wiley, 1981.

[11] T. Kailath, L. Ljung, and M. Morf, "Generalized Krein-Levinson equations for efficient calculation of Fredholm resolvents of nondisplacement kernels," in Topics in Functional Analysis, (Essays in Honor of M. G. Krein), Advances in Mathematics Supplementary Studies, vol. 3, I. Gohberg and M. Kac Eds. New York: Academic, 1978, pp. 169-184.

[12] L. Lapidus and G. F. Pinder, Numerical Solution of Partial Differential Equations in Science and Engineering. New York: Wiley, 1982.

[13] M. M. Crum, "Associated Sturm-Liouville systems," Quart. J. Math., vol. 6, no. 2, pp. 121-128, 1955.

[14] M. G. Krein, "The continuous analogue of a formula of Christoffel in the theory of orthogonal polynomials," Dokl. Akad. Nauk. SSSR, vol. 113, no. 5, pp. 970-973, 1957.

[15] Z. S. Agranovich and V. A. Marchenko, The Inverse Problem of Scattering Theory. New York: Gordon and Breach, 1963.

[16] P. Deift and E. Trubowitz, "Inverse scattering on the line," Commun. Pure Appl. Math., vol. 32, no. 2, pp. 121-251, 1979.

[17] I. M. Gelfand and B. M. Levitan, "On the determination of a differential equation by its spectral function," Izv. Akad. Nauk SSSR, Math. Ser., vol. 15, pp. 309-360, 1951 (Transl.: Amer. Math. Soc. Transl., ser. 2, vol. 1, pp. 253-304, 1955).

[18] K. Chadan and P. C. Sabatier, Inverse Problems in Quantum Scattering Theory. New York: Springer-Verlag, 1977.

[19] L. D. Faddeev, "The inverse problem in the quantum theory of scattering," J. Math. Phys., vol. 4, no. 1, pp. 72-104, Jan. 1963.

[20] E. Wong, Stochastic Processes in Information and Dynamical Systems. New York: McGraw-Hill, 1971. 